\title{
High-resolution genetic mapping with pooled sequencing
}

\author{
Matthew D Edwards ${ }^{1}$, David K Gifford ${ }^{1,2,3^{*}}$ \\ From Second Annual RECOMB Satellite Workshop on Massively Parallel Sequencing \\ Barcelona, Spain. 19-20 April 2012
}

\begin{abstract}
Background: Modern genetics has been transformed by high-throughput sequencing. New experimental designs in model organisms involve analyzing many individuals, pooled and sequenced in groups for increased efficiency. However, the uncertainty from pooling and the challenge of noisy sequencing data demand advanced computational methods.
\end{abstract}

Results: We present MULTIPOOL, a computational method for genetic mapping in model organism crosses that are analyzed by pooled genotyping. Unlike other methods for the analysis of pooled sequence data, we simultaneously consider information from all linked chromosomal markers when estimating the location of a causal variant. Our use of informative sequencing reads is formulated as a discrete dynamic Bayesian network, which we extend with a continuous approximation that allows for rapid inference without a dependence on the pool size. MULTIPOOL generalizes to include biological replicates and case-only or case-control designs for binary and quantitative traits.

Conclusions: Our increased information sharing and principled inclusion of relevant error sources improve resolution and accuracy when compared to existing methods, localizing associations to single genes in several cases. MULTIPOOL is freely available at http://cgs.csail.mit.edu/multipool/.

\section{Background}

Advances in high-throughput DNA sequencing have created new avenues of attack for classical genetics problems. A robust method for determining the genetic elements that underlie a phenotype is to gather and group individuals of different phenotypes, interrogate the genome sequences of each group, and identify elements that are present in different proportions between the groups. We describe MULTIPOOL, a multi-locus method for analyzing high-throughput DNA sequencing reads obtained from large pools of phenotypicallyextreme individuals.

\footnotetext{
* Correspondence: gifford@mit.edu

${ }^{1}$ Computer Science and Artificial Intelligence Laboratory, Department of Electrical Engineering and Computer Science, Massachusetts Institute of Technology, Cambridge, MA 02139, USA

Full list of author information is available at the end of the article
}

\section{Targeted experiments}

We focus on model organism experiments where two strains are crossed and the progeny are grouped and pooled according to phenotype. We describe and model experiments for haploid organisms that are hybrids between two strains, but we note that the models we develop should generalize to more sophisticated crosses or diploid organisms. When two strains vary in a phenotype, analyzing progeny with extreme phenotypes should elucidate the genetic basis of the trait. The main idea is that polymorphic loci that do not affect the phenotype will segregate with approximately equal frequency in the progeny (regardless of phenotype), while loci that influence the trait will be enriched in opposite directions in the extreme individuals, according to the effect size of each locus. This approach assumes that the causal loci have sufficiently strong main effects to be detectable via any type of pooled analysis. This pooled study design is also referred to as "bulk 
segregant analysis" [1] in model system genetics. Selection and pooling based on a quantitative phenotype can identify quantitative trait loci (QTLs), so this procedure can also be viewed as a type of pooled QTL mapping. Figure 1 illustrates the experimental design at a broad level, though there are many ways to design crosses and experimental selections to produce pools that may be analyzed by MULTIPOOL.
Bulk segregant analysis with high-throughput sequencing has been applied in yeast to study drug resistance in [2], high temperature growth in [3], and viability on alternate carbon sources in [4]. Related pooled sequencing experiments used fly [5] and Arabidopsis [6] model systems. In human, analogous pooled sequencing studies currently require target capture methods and a preselected set of candidate loci [7].

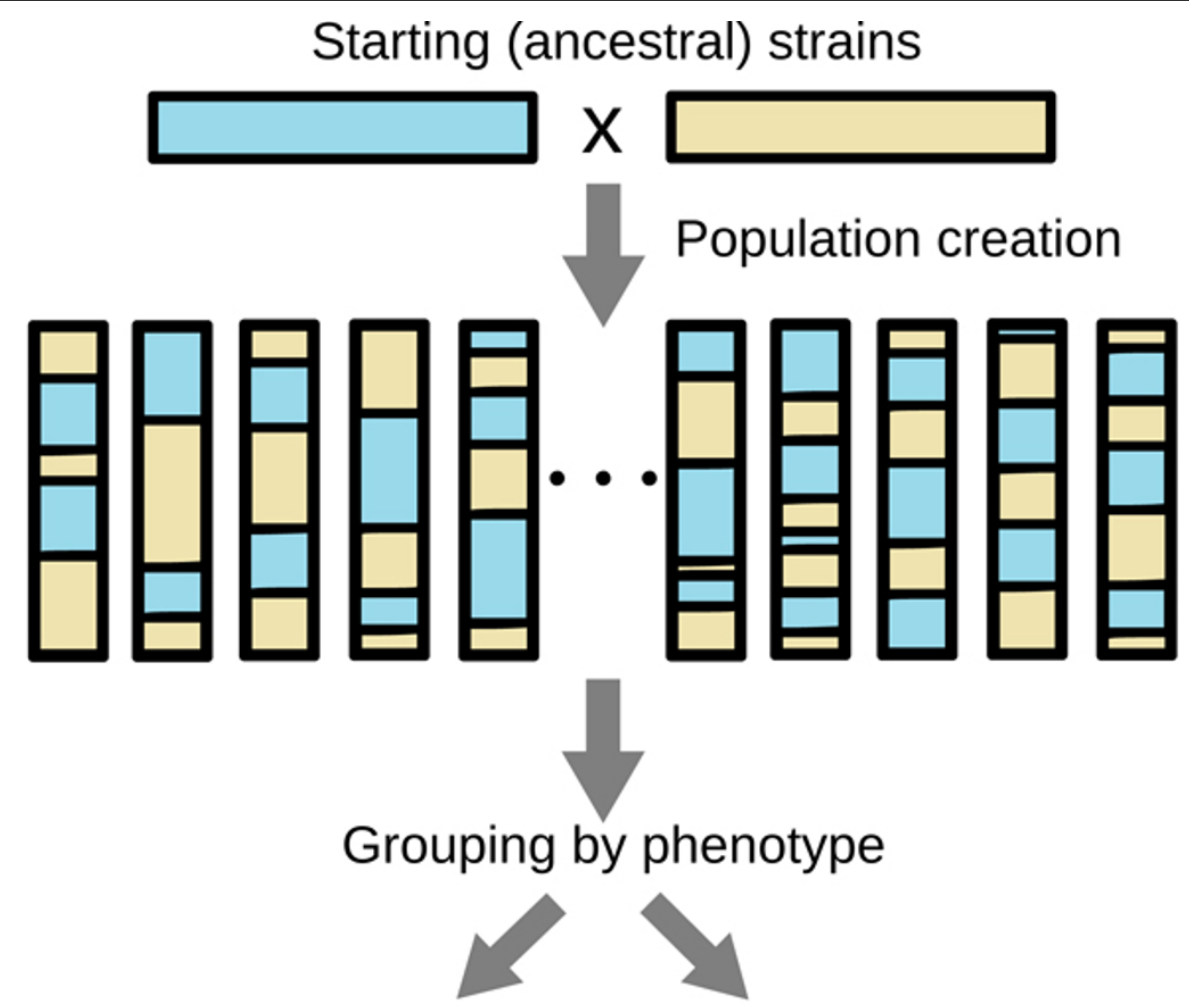

Pooled high-throughput sequencing of groups

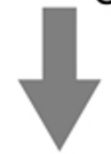

Allele frequency measurements in pools
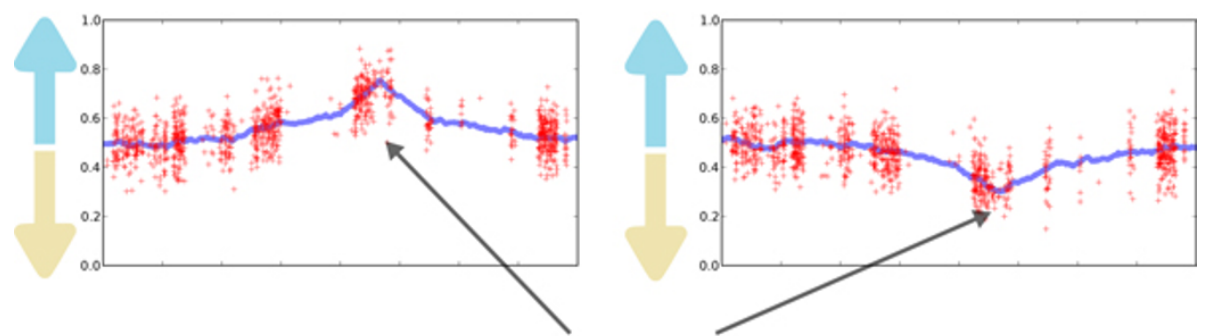

Causal locus (QTL) identification

Figure 1 Experimental design example. Strains are crossed and hybrid progeny are collected. The progeny are grouped by phenotype and the pooled DNA of each group is subjected to high-throughput DNA sequencing. Loci that affect the phenotype show an enrichment for one strain in each pool, while other unlinked loci segregate evenly. The bottom two plots show simulated (unobserved) allele frequencies in the pool with blue lines and (observed) allele frequencies computed from simulated 50X sequencing coverage in red. 
Pools may be selected from a single phenotypic extreme, opposite extremes, or one extreme and a control sample. Pools may also be obtained by grouping based on binary traits rather than quantitative phenotype extremes. Early studies used microarrays for pooled genotyping [8-10], but recent developments in highthroughput sequencing allow its use as a direct genotyping platform. While genotyping or sequencing individuals is an alternate choice, the appeal of pooled analysis is the dramatic reduction in cost while maintaining the statistical power of large sample sizes. See [11-13] for a discussion on pooled association studies and experiment design considerations.

\section{Challenges}

Pooled genetic mapping studies using high-throughput sequencing present a number of unique difficulties. The core statistical quantity of interest, the allele frequency in each pool, is observed only indirectly. The strain-specific read counts that are used to estimate the allele frequencies are corrupted by sampling noise at most reasonable sequencing depths, read mapping errors [14], reference genome inaccuracies, and biological bias during sample preparation. In addition, the allele frequency measurements are nonuniformly spaced along the genome, depending on the polymorphism structure between the strains of interest. As an illustration, we refer to the bottom two plots in Figure 1 which show simulated 50X average sequencing coverage using polymorphisms from two yeast strains. Linkage implicates a wide region along the shown chromosome, and the allele frequencies estimated from read counts are noisy and not necessarily highest at the exact location of the causal allele.

However, the unbiased nature of genotyping via highthroughput sequencing results in nearly saturated marker coverage where almost all polymorphisms are queried. This avoids the laborious process of marker discovery and assay design required by earlier genotyping technologies. The dense marker coverage also allows for a high degree of information sharing, which motivates the methods underlying MULTIPOOL.

\section{Previous statistical methods}

Previous statistical approaches to analyzing pooled genotyping data have focused on alternate regimes where genetic markers are relatively sparse and measurements are relatively accurate. Often, only single loci are tested for association, necessarily ignoring data from nearby markers. Additionally, single-locus methods encounter difficulties with missing data, such as regions that are difficult to sequence or map or have very few polymorphisms.

Earlier work applied hidden Markov models (HMMs) to fine mapping within small regions with fewer number of markers [15,16], and was extended to pooled genotype measurements in similar scenarios [17]. However, these methods relied on computationally intensive sampling methods and were applied to datasets with only a few dozen markers. Conceptually similar methods have been explored for human studies, focusing on utilizing haplotype structure in the analysis of pooled experiments [18]. In more recent pooled sequencing experiments, a slidingwindow method was applied on $p$-values from local tests in [2], while a local weighted method motivated by a probabilistic model was given in [3]. However, these models do not explicitly model the location of the causal locus while considering all relevant marker data.

\section{Approach}

MULTIPOOL is designed for experimental crosses and dense noisy genotyping, as obtained by sequencing, and handles datasets with tens or hundreds of thousands of markers. We develop a statistical model that can combine information across many nearby markers while accounting for the nonuniform noise levels introduced by varying sequencing depth and marker spacing. The specific advances we present with MULTIPOOL include:

- A model-based framework that allows for information sharing across genomic loci and incorporation of experiment-specific noise sources. These methods improve on previous approaches that rely on heuristic techniques to select sliding window sizes, which may sacrifice resolution.

- Statistical tests using an information-sharing dynamic Bayesian network (DBN) that report robust location estimates and confidence intervals. The multi-locus methods allow for principled inference even in regions without strain-specific markers and reduce experimental noise when many markers are available.

- Extensions of our method to any number of replicates and multiple experimental designs, within the same principled statistical framework.

\section{Methods}

We develop inference methods for the pool allele frequency at a particular genome position, given the pooled read samples. First, we propose generative models which describe the experimental process. Next, these models are used to construct likelihood-based statistics to assess the significance of associations in multiple experimental designs.

\section{Obtaining allele frequency measurements}

All sequencing reads from a particular pooling experiment are aligned to one strain's reference genome using 
the short read aligner bwa[19]. To increase specificity, only uniquely-mapping reads are considered. In practice, any short read aligner that can produce or export its output to the standard SAM format is compatible with this workflow. Next, a whole-genome pileup is generated using samtools[20]. A genome pileup lists the particular base calls at each genomic position, using the set of mapped sequencing reads. The genome pileup produces reference and non-reference allele counts at each base. Using single-strain sequencing data, lists of polymorphic bases can be determined and extracted from the pileup of the pooled experiments. The result is a list of allelespecific read counts at many polymorphic sites across the genome. The coverage of the marker sites will vary according to local sequencing depth and mappability [14], and the density will vary according to the local polymorphism level. A similar approach was applied to generate allele counts in [2].

\section{Multi-locus model}

MULTIPOOL uses a probabilistic model that considers one chromosome at a time and explicitly models the effect that recombination and pool size have on neighboring allele frequencies. The model is a dynamic Bayesian network that describes the changing allele frequencies in the pool along a chromosome. The chromosome of interest is segmented into discrete blocks of equal size. A hidden state corresponding to each block reflects the pool allele frequency in the pool at that locus, varying along the genome as recombination causes random fluctuations. Each locus may emit sequencing reads according to its local pool allele frequency (hidden state). These reads may originate from multiple markers falling within the same region or a single marker. When there are no polymorphisms or mappable reads available in a region, the locus has no emissions and therefore the observed data do not directly constrain the hidden state at that locus. Finally, a particular locus may include the causal gene and therefore be directly associated with the phenotype. We assume there is only one causal locus in the analyzed region. For the genetic mapping problem, the causal locus is unknown and the key inference task is identifying its location and degree of association with the phenotype.

\section{Model specification}

The pool is composed of $N$ individuals. An unknown causal locus is linked to the phenotype and displays association with allele frequency $p \neq \frac{1}{2}$ in the population. Loci that are not associated with the phenotype and are not linked to the causal locus segregate at frequency $p=\frac{1}{2}$ in the population. The pool allele frequencies are unobserved and are given for each genome segment $i$ by $x_{i}, i=\{1, .$. , $L\}$. The observed allele frequency measurements $y_{i}$ are obtained from the mapped sequencing reads. We also define $d_{i}$, the total informative reads at each locus. This quantity is determined by the local sequencing depth and number of mappable polymorphisms. The recombination frequency $r$ gives the probability of an odd number of crossovers between adjacent genome segments in one individual in the pool. We do not model crossover interference, and therefore assume that recombination events are independent along the genome. The dependencies encoded in this model can be expressed as a graphical model, shown in Figure 2. While the example figure shows a particular choice of the causal locus, the inference task consists of selecting among all possible choices (model structures) for the causal locus and the population allele frequency $p$. The population allele frequency is the allele

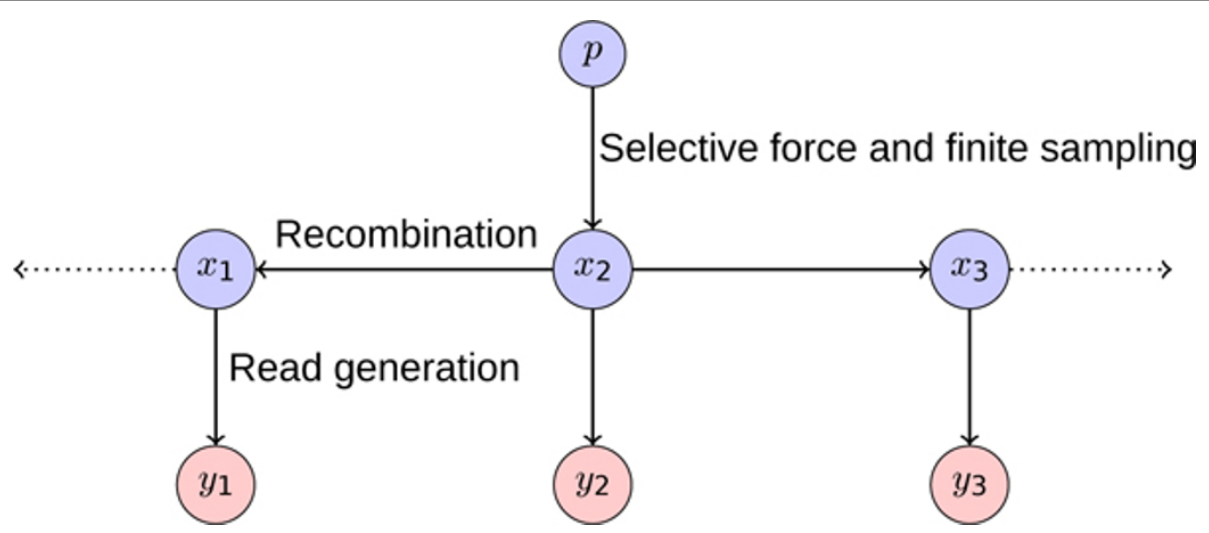

Figure 2 Graphical model showing multi-locus dependencies. Dynamic Bayesian network used by MULTIPOOL to capture the dependence between nearby loci in a pooled sequencing experiment. Allele frequencies in the pool influence the mix of observed sequencing reads at each locus. Here, the causal allele is $x_{2}$ and its value is determined by sampling $N$ individuals to create the pool from the population allele frequency $p$ (where $p \neq \frac{1}{2}$ indicates association). 
frequency of the causal locus that would be observed in an infinitely-large pool (the population), and depends on the strength of the locus's association. Subsequent sections develop efficient methods for calculating likelihoods for all relevant model structures by reusing intermediate computations.

\section{Emission probabilities}

The probability of observing a set of sequencing reads conditioned on the pool fraction at the locus and a total informative read count $d_{i}$ can be calculated using the binomial distribution:

$$
y_{i} \cdot d_{i} \sim \operatorname{Bin}\left(d_{i}, x_{i}\right)
$$

This formulation models the read count proportion exactly with a discrete model. An approximation, applicable to high read counts, can be obtained with a Gaussian distribution:

$$
y_{i} \sim \mathcal{N}\left(x_{i} \frac{x_{i}\left(1-x_{i}\right)}{d_{i}}\right) .
$$

Technical pooling variance that increases the local measurement noise, such as allele-specific PCR amplification bias, could be assumed to act in locus-independent manner and be modeled with increased variance in this expression.

\section{Transition probabilities}

In practice, the genome segments are chosen to be small enough so that $r$ is effectively the probability of a single recombination event occurring. We can determine the transition probabilities from $x_{i}$ to $x_{i+1}$ by considering the $k$ individuals that switch from the first strain to the second and the $j$ individuals of the reverse case. We know $k \sim \operatorname{Bin}\left(N x_{\mathrm{i}}, r\right)$ since each of the $N x_{i}$ individuals with the first strain's ancestry at locus $i$ will switch strain type when a recombination event occurs, with probability $r$. Similarly, $j \sim \operatorname{Bin}\left(N\left(1-x_{i}\right), r\right)$. Thus:

$$
x_{i+1}=x_{i}-\frac{k}{N}+\frac{j}{N} .
$$

Employing normal approximations for the binomial distributions and dividing by $N$, we obtain an approximation for the transition probabilities:

$$
\begin{aligned}
& x_{i+1} \sim x_{i}-\mathcal{N}\left(x_{i} r, \frac{x_{i} r(1-r)}{N}\right)+\mathcal{N}\left(\left(1-x_{i}\right) r, \frac{\left(1-x_{i}\right) r(1-r)}{N}\right) \\
& =\mathcal{N}\left(x_{i}(1-2 r)+r, \frac{(1-r) r}{N}\right) .
\end{aligned}
$$

This formulation shows that the latent allele frequencies form a first-order autoregressive Gaussian process with mean $\frac{r}{1-(1-2 r)}=\frac{1}{2}$ and variance $\frac{(1-r) r / N}{1-(1-2 r)^{2}}=\frac{1}{4 N}$, which can be verified with a single-locus analysis.

\section{Initial probabilities}

The causal locus node induces a particular distribution over hidden states, depending on the selected population allele frequency $p$ :

$$
x_{i} \cdot N \sim \operatorname{Bin}(N, p) .
$$

The normal approximation is:

$$
x_{i} \sim \mathcal{N}\left(p, \frac{p(1-p)}{N}\right) .
$$

\section{Inference: discrete model}

Inference of the hidden state values can proceed outwards from the causal locus, using the conditional independence structure of the model. We describe the algorithms in terms of standard HMM techniques, but note that a more general treatment in terms of message passing is also possible.

The observed data likelihood $\operatorname{Pr}(\vec{y})$, conditioned on a particular causal allele at $x_{c}$ (model structure) and population allele frequency $p$, is obtained by conditioning on the values of the causal locus:

$$
\operatorname{Pr}(\vec{y} \mid p)=\sum_{j=0}^{N} \operatorname{Pr}\left(\vec{y}_{(c+1, \ldots, L)} \mid x_{c}=\frac{j}{N}\right) \operatorname{Pr}\left(\vec{y}_{(1, \ldots, c-1)} \mid x_{c}=\frac{j}{N}\right) \operatorname{Pr}\left(y_{c} \mid x_{c}=\frac{j}{N}\right) \operatorname{Pr}\left(x_{c}=\frac{j}{N} \mid p\right) .
$$

The first term in the sum operates on an HMM with rightwards arrows in its graph, while the second term operates on an HMM with leftwards arrows (see Figure 2). However, the latent states form a reversible Markov chain, allowing us to reverse the arrows in the left graphical model fragment. After this transformation, the likelihood computations for all choices of the causal node $x_{c}$ use the same graphical structure over the latent states $\vec{x}$ when conditioned on the causal node $x_{c}$ : two chains with all rightwards arrows, separated by the conditioned node $x_{c}$. Using this fact, we can compute the desired likelihoods with intermediate computations from a single graphical model.

We compute the product of the first three terms in the sum, $\operatorname{Pr}\left(\vec{y} \mid x_{c}\right)$, using the posterior distribution of $x_{c}$ computed using an HMM with no causal locus $\left(\operatorname{Pr}\left(x_{c} \mid \vec{\gamma}\right)\right)$. The posterior distributions are calculated using the forward-backward algorithm [21,22], using the transition and emission distributions given previously. The unconditional marginal distribution $\operatorname{Pr}\left(x_{c}\right)$ is computed using the stationary distribution of the latent allele frequencies in the noncausal model.

$$
\operatorname{Pr}(\vec{\gamma} \mid p)=\sum_{j=0}^{N} \operatorname{Pr}\left(\vec{\gamma} \mid x_{c}=\frac{j}{N}\right) \operatorname{Pr}\left(x_{c}=\frac{j}{N} \mid p\right)=\operatorname{Pr}(\vec{\gamma}) \sum_{j=0}^{N} \frac{\operatorname{Pr}\left(x_{c}=\frac{j}{N} \mid \vec{\gamma}\right)}{\operatorname{Pr}\left(x_{c}=\frac{j}{N}\right)} \operatorname{Pr}\left(x_{c}=\frac{j}{N} \mid p\right)
$$

Running the forward-backward algorithm requires considering all transitions in each chromosome block, leading to a runtime quadratic in the size of the pool: $O\left(N^{2} L\right)$. This dominates the cost for the final step of computing 
$\operatorname{Pr}(\vec{y} \mid p)$ for all causal locus locations and a fixed $p$, which is $O(N L)$. The quadratic dependence on the pool size renders the exact modeling of large pools prohibitive, motivating the continuous approximation in the next section.

\section{Inference: continuous approximation}

The previous inference procedure applied to discrete hidden states where the pool composition is modeled exactly, but yielded inference algorithms that require time quadratic in the size of the pool. For large pools, we can relax this requirement and avoid the quadratic burden by modeling the allele frequency as a continuous value. The graphical model is linear-Gaussian since the transitions and observations are linear functions of the latent variables, subject to Gaussian noise. In a linear dynamical systems formulation, the model is:

$$
\begin{gathered}
x_{i+1}=x_{i}-x_{i} r+\left(1-x_{i}\right) r+w=(1-2 r) x_{i}+r+w, \\
y_{i}=x_{i}+v_{i} .
\end{gathered}
$$

Where:

$$
\begin{gathered}
w \sim \mathcal{N}\left(0, \frac{(1-r) r}{N}\right), \\
v_{i} \sim \mathcal{N}\left(0, \frac{1}{4 d_{i}}\right) .
\end{gathered}
$$

The per-locus observation noise $v_{i}$ can be approximated with the sample variance from the observed $y_{i}$, depending on $y_{i}$ and $d_{i}$, or upper bounded by $\frac{1}{4 d_{i}}$. The posterior probabilities over the continuous latent $i_{\text {states }}$ can be calculated with the Kalman filtering and smoothing equations, analogous to the two recursive functions used to calculate the posterior probabilities for HMMs [21-23]. The Kalman filtering equations yield the conditional distribution of the latent state given the preceding observations with a recursive estimate:

$$
\begin{gathered}
\operatorname{Pr}\left(x_{i} \mid \vec{\gamma}_{\{1, \ldots, i\}}\right)=\mathcal{N}\left(x_{i} ; \mu_{i}, \sigma_{i}^{2}\right), \\
\mu_{i}=(1-2 r) \mu_{i-1}+r+K_{i}\left(y_{i}-(1-2 r) \mu_{i-1}-r\right), \\
\sigma_{i}^{2}=\left(1-K_{i}\right) P_{i-1} .
\end{gathered}
$$

Where:

$$
\begin{gathered}
P_{i-1}=(1-2 r)^{2} \sigma_{i-1}^{2}+\frac{r(1-r)}{N}, \\
K_{i}=\frac{P_{i}-1}{P_{i-1}+\frac{1}{4 d_{i}}} .
\end{gathered}
$$

The recursions begin with the stationary distribution parameters:

$$
\begin{aligned}
\mu_{0} & =\frac{1}{2}, \\
\sigma_{0}^{2} & =\frac{1}{4 N}, \\
P_{0} & =\frac{1}{4 N} .
\end{aligned}
$$

The Kalman smoothing equations use the filtered results (forward estimates) to create estimates of the hidden state using the entire observation sequence, recursing backwards:

$$
\begin{gathered}
\operatorname{Pr}\left(x_{i} \mid \vec{\gamma}\right)=\mathcal{N}\left(x_{i} ; \hat{\mu}_{i}, \hat{\sigma}_{i}^{2}\right), \\
\left.\hat{\mu}_{i}=\mu_{i}+J_{i} \hat{\mu}_{i+1}-(1-2 r) \mu_{i}-p\right), \\
\hat{\sigma}_{i}^{2}=\sigma_{i}^{2}+J_{i}^{2}\left(\hat{\sigma}_{i+1}^{2}-P_{i}\right) .
\end{gathered}
$$

Where:

$$
\begin{gathered}
J_{i}=\frac{\sigma_{i}^{2}(1-2 r)}{P_{i}}, \\
\hat{\mu}_{L}=\mu_{L}, \\
\hat{\sigma}_{L}^{2}=\sigma_{L}^{2} .
\end{gathered}
$$

As in the discrete section, the posterior distributions of the latent states under a null model can be used to compute the desired data likelihoods for all possible causal models. Required integrals are computed numerically using a fixed number of points. Specifically:

$$
\operatorname{Pr}(\vec{\gamma} \mid p)=\int_{0}^{1} \operatorname{Pr}\left(\vec{\gamma} \mid x_{c}=j\right) \operatorname{Pr}\left(x_{c}=j\right) \mathrm{d} j=\operatorname{Pr}(\vec{\gamma}) \int_{0}^{1} \frac{\operatorname{Pr}\left(x_{c}=j \mid \vec{y}\right)}{\operatorname{Pr}\left(x_{c}=j\right)} \operatorname{Pr}\left(x_{c}=j \mid p\right) \mathrm{d} j .
$$

Since the probability distributions during inference are represented with a constant number of parameters instead of a full vector (as in the discrete case), inference is more efficient. Specifically, computing the required quantities $\operatorname{Pr}\left(x_{c}=j \mid \vec{y}\right)$ for all $c$ requires $O(L)$ time. This removes the dependence on the size of the pool that was present in the discrete method, allowing MultiPool to perform accurate inference in very large pools.

\section{Statistical tests}

With these computations in place, we can compare all values of the causal locus and the trait association, measured by $p$. For each locus, we construct a likelihood ratio statistic comparing the hypotheses of association and no association:

$$
L R(c)=\frac{\max _{p^{\prime}} \operatorname{Pr}\left(\vec{y} \mid p=p^{\prime}\right)}{\operatorname{Pr}\left(\vec{y} \mid p=\frac{1}{2}\right)}=\max _{p^{\prime}} \sum_{j=0}^{N} \frac{\operatorname{Pr}\left(x_{c}=\frac{j}{N} \mid \vec{y}\right)}{\operatorname{Pr}\left(x_{c}=\frac{j}{N}\right)} \operatorname{Pr}\left(x_{c}=\frac{j}{N} \mid p=p^{\prime}\right) .
$$

The simplification occurs because the likelihood under the noncausal hypothesis at any locus is the same, namely $\operatorname{Pr}(\vec{y})$ from the noncausal HMM. A similar likelihood is obtained with the continuous model:

$$
L R(c)=\max _{p^{\prime}} \int_{0}^{1} \frac{\operatorname{Pr}\left(x_{c}=j \mid \vec{\gamma}\right)}{\operatorname{Pr}\left(x_{c}=j\right)} \operatorname{Pr}\left(x_{c}=j \mid p=p^{\prime}\right) \mathrm{d} j .
$$

We perform the maximization over $p$ ' numerically and calculate the likelihood ratio for all positions of the causal locus by reweighting the posterior probabilities. 


\section{Multiple experiments}

We can analyze replicate experiments by forming a coupled dynamic Bayesian network. This analysis present two replicates, but the methods generalize to any number of coupled experiments. In this situation, the same sampling distribution is induced at the shared causal locus in two coupled chains. The joint data likelihood factors since the chains are conditionally independent given the selection node $p$ :

$$
L R(c)=\max _{p^{\prime}} \frac{\operatorname{Pr}\left(\vec{y}_{1}, \vec{y}_{2} \mid p=p^{\prime}\right)}{\operatorname{Pr}\left(\vec{y}_{i}, \vec{\gamma}_{2} \mid p=\frac{1}{2}\right)}=\frac{\max _{p^{\prime}} \operatorname{Pr}\left(\vec{\gamma}_{1} \mid p=p^{\prime}\right) \operatorname{Pr}\left(\vec{\gamma}_{2} \mid p=p^{\prime}\right)}{\operatorname{Pr}\left(\vec{\gamma}_{i}\right) \operatorname{Pr}\left(\vec{\gamma}_{2}\right)} .
$$

The maximization over $p^{\prime}$ must consider the product of the data likelihoods in the replicates. For designs where paired experiments are expected to show opposite effects, each experiment selects an optimal population allele frequency $p$. In this case, the null hypothesis is the coupled model where the two experiments share the same population allele frequency. The likelihood ratio is:

$$
L R(c)=\frac{\max _{p_{1}, p_{2}} \operatorname{Pr}\left(\vec{\gamma}_{i} \mid p=p_{1}\right) \operatorname{Pr}\left(\vec{\gamma}_{2} \mid p=p_{2}\right)}{\max _{p_{3}} \operatorname{Pr}\left(\vec{\gamma}_{1}, \vec{\gamma}_{2} \mid p=p_{3}\right)}=\frac{\max _{p_{1}} \operatorname{Pr}\left(\vec{\gamma}_{1} \mid p=p_{1}\right) \max _{p_{2}} \operatorname{Pr}\left(\vec{\gamma}_{2} \mid p=p_{2}\right)}{\max _{p_{3}} \operatorname{Pr}\left(\vec{\gamma}_{1} \mid p=p_{3}\right) \operatorname{Pr}\left(\vec{\gamma}_{2} \mid p=p_{3}\right)} .
$$

The numerator is the product of two single-experiment maximizations, while the denominator is the coupled model likelihood that was presented for replicate analysis.

Using these results, MultiPool reports $\log _{10}$ likelihood ratios (LOD scores in the genetics community), maximum-likelihood estimates (MLE) of the causal locus location, and approximate credible intervals for the location of the causal locus. Assuming a uniform prior over causal locus locations, $\operatorname{Pr}\left(x_{c} \mid \vec{y} \propto \operatorname{Pr}\left(\vec{y} \mid x_{c}\right)\right.$ for a particular set of observations $\vec{\gamma}$. In each case we fix $p$ at its MLE, but could alternately integrate it out. Therefore, we can compute multi-locus statistics that include information from the entire dataset in experiments where multiple pools are available.

\section{Results}

\section{Simulation results}

In order to understand the benefit of MultiPool versus single-locus tests on deeply-sequenced pools, we conducted a series of simulations. A causal locus was chosen with population allele frequency $p=0.75$ and many pools of sizes $N=100,1000$, and 10000 were created. SNP locations and relative per-SNP sequencing depths were calculated from experimental datasets in yeast. Average read coverage (sequencing depth) was varied from $10 \mathrm{X}$ to $150 \mathrm{X}$, and 100 datasets of each type were simulated. The MLE causal allele location was calculated with the single-pool DBN model. The single-locus test analyzed allele frequencies computed with $1 \mathrm{~kb}$ sliding windows, based on the method in [12]. The root mean square errors for each pool size and sequencing depth is shown in Figure 3. In the $N=100$ cases, the mapping accuracy is predominantly controlled by the small pool size. This leads to little improvement with increased sequencing depth. The larger pools show higher accuracy with increased sequencing depth, but MultiPool is always more accurate with a lower sequencing requirement. These simulations were conducted without additional read mapping noise or other noise sources, and so the absolute results should be interpreted conservatively.

\section{Experimental results}

We also analyze pooled sequencing data recently generated by two groups $[2,3]$. The groups generated haploid yeast individuals with hybrid backgrounds from two strains and performed various phenotypic selections. Table 1 lists the datasets and their sequencing depths. While each experiment generated many statistically significant novel results, we limit ourselves to mapping comparisons involving target genes that have been validated using targeted follow-up experiments. We note that even though a target gene may be verified as affecting the trait, an untested nearby gene may affect the localization results.

\section{Single-locus comparisons}

In cases where the associated region is localized to a single gene, we compare the LOD scores from MultiPool to a likelihood ratio computed using allele frequencies calculated by summing allele read counts in sliding windows. The data likelihoods under the causal and noncausal models are calculated according to the model in [12], with the genotyping noise calculated from the local informative read depth. We use 50-bp genome segments in the dynamic Bayesian network (DBN) model and set the recombination rate in the model to the empirical average in yeast [24].

\section{Large pool results}

The first set of large pools was used to characterize the genetic basis of resistance to the DNA-damaging agent 4-NQO. The genes RAD5 and MKT1 were validated as affecting 4-NQO resistance with follow-up experiments, so we use them as test cases for our model. The control pools showed no association around the validated loci, so we applied MultiPool's one-pool test for association using the continuous model. Table 2 shows the distances from the MLE peak estimate to the middle of the target gene from MultiPool and sliding-window tests.

MultiPool localizes $R A D 5$ to within the gene body, without a dependence on choosing an appropriate sliding window size. The $90 \%$ credible interval of the location contains six genes, centered on RAD5. A localization example using one replicate is shown in Figure 4. MKT1 is localized to within $3 \mathrm{~kb}$ of the gene 


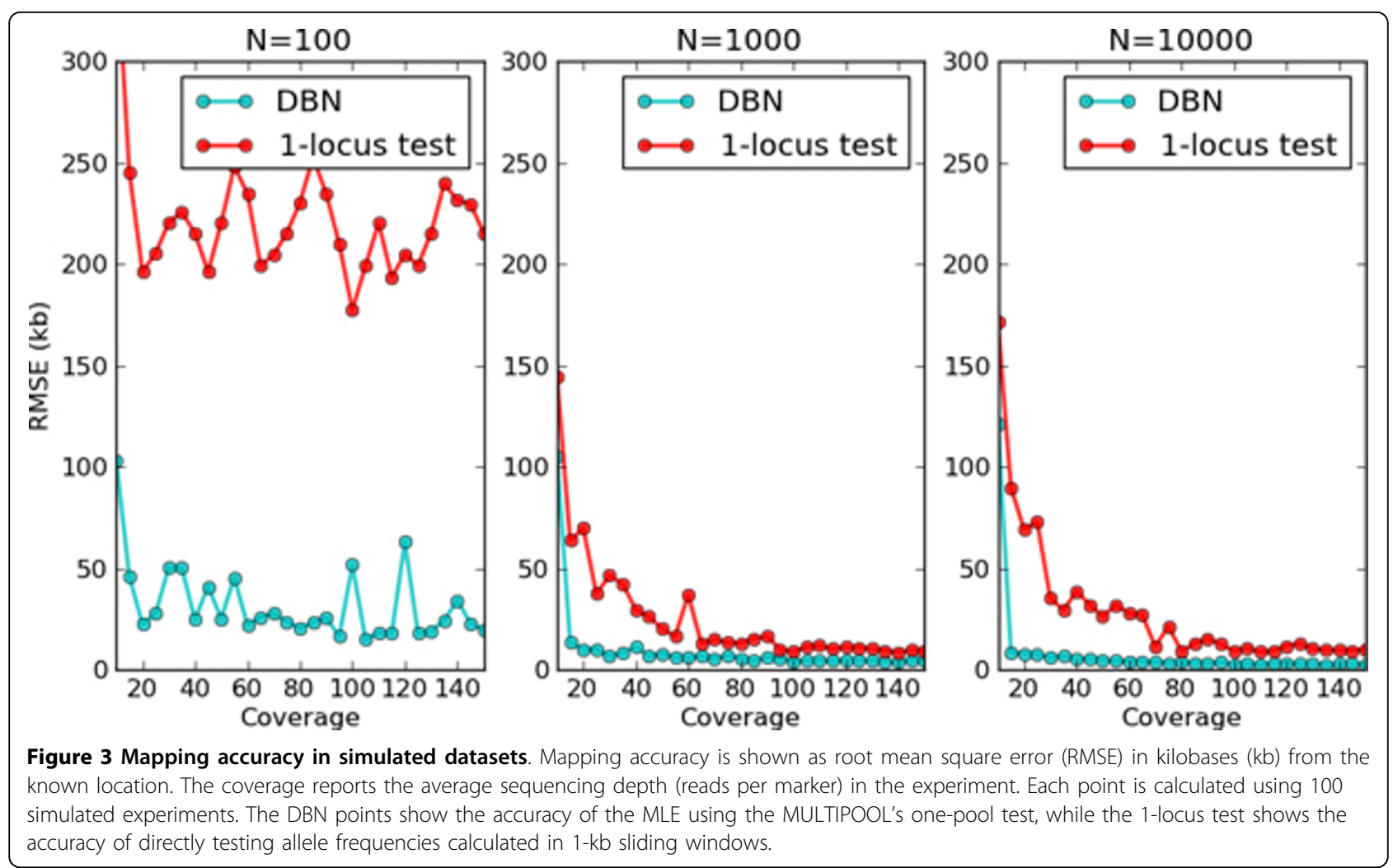

body, with the $90 \%$ credible interval covering $M K T 1$ and eight other genes.

The second set of large pools was constructed to study the genetics of heat tolerance, using repeated crosses to reduce linkage disequilibrium (increased $r$ in our model). In this study, the genes IRA1 and IRA2 were verified as affecting heat sensitivity with direct assays. Table 3 reports the distance from the MLE estimate to the center of the target gene using MultiPool and sliding window methods. IRA2 is localized to within the gene body, but the predictions for IRA1 are consistently upstream of the gene's location. Upon further investigation, the peak around IRA1 appears to contain another (untested) associated locus. Figure 5 plots the estimated allele frequencies and LOD scores in the surrounding region. The relevant $90 \%$ credible intervals for the causal locus location include IRA2 in all datasets, but do not include IRA1. This may support the hypothesis of another linked gene in the associated region.

\section{Conclusion}

We presented MultiPool, a computational method to map genetic elements from pooled sequencing studies. Taking advantage of recent increases in throughput, these experimental designs use sequencing to provide unbiased and labor-efficient genotyping. As throughput continues to increase, similar studies will be extended to larger and more complex genomes. By including all relevant data in a unified framework, MultiPool improves the analysis of these experiments with increased accuracy and the principled estimation of association intervals. The statistical framework is most beneficial for the case where there are many noisy markers, as observed in genotyping via sequencing. In these cases, combining

Table 1 Analyzed experiments

\begin{tabular}{|c|c|c|c|c|c|}
\hline Name & Read length & Pool size & Coverage (rep. 1) & Coverage (rep. 2) & Source (ref.) \\
\hline 4-NQO viable & 76 & $\approx 10000$ & 67.7 & 85.0 & [2] \\
\hline Control & 76 & $\approx 10000$ & 36.1 & 79.5 & [2] \\
\hline Heat tolerant & 76 (paired) & $\approx 10000$ & 152.4 & 84.8 & [3] \\
\hline Control & 76 (paired) & $\approx 10000$ & 79.0 & 75.2 & [3] \\
\hline
\end{tabular}

Each condition was assayed with two replicates. Coverage is the average reads per marker. Due to the protocols used, precise quantification of the pool size is difficult. We used the listed values as conservative choices since the reported ranges are larger in most cases. 
Table 2 Localization of known associated genes in large drug-selected pools

\begin{tabular}{lcccc}
\hline Dataset & Target & $\begin{array}{c}\text { DBN } \\
\text { dist. }\end{array}$ & $\begin{array}{c}\text { 1kb window } \\
\text { dist. }\end{array}$ & $\begin{array}{c}\text { 10kb window } \\
\text { dist. }\end{array}$ \\
\hline $\begin{array}{l}\text { 4-NQO viable } \\
\text { rep. 1 }\end{array}$ & RAD5 & 5305 & 18355 & 14605 \\
$\begin{array}{l}\text { 4-NQO viable } \\
\text { rep. 2 }\end{array}$ & RAD5 & 745 & 6195 & 3145 \\
\hline Combined & RAD5 & 805 & 755 & 3145 \\
\hline $\begin{array}{l}\text { 4-NQO viable } \\
\text { rep. 1 }\end{array}$ & MKT1 & 3223 & 15127 & 1673 \\
$\begin{array}{l}\text { 4-NQO viable } \\
\text { rep. 2 }\end{array}$ & MKT1 & 5223 & 15127 & 5423 \\
\hline $\begin{array}{l}\text { Combined } \\
\text { MKT1 }\end{array}$ & 4323 & 15127 & 5423
\end{tabular}

Distances are reported in bases from the MLE to the center of the target gene.

information across the genome is critical in reducing noise and increasing statistical power. More generally, the methods developed and applied in this work support the application of selection and pooled genotyping for experimental organisms. When experimental procedures can create medium or large allele frequency differences, the responsible genes can be mapped with great precision. These methods do not require the step of explicit polymorphism discovery or genotyping array design, yielding large time and cost savings.
Table 3 Localization of known associated genes in large heat-selected pools

\begin{tabular}{lcccc}
\hline Dataset & Target & $\begin{array}{c}\text { DBN } \\
\text { dist. }\end{array}$ & $\begin{array}{c}\text { 1 kb window } \\
\text { dist. }\end{array}$ & $\begin{array}{c}\text { 10kb window } \\
\text { dist. }\end{array}$ \\
\hline $\begin{array}{l}\text { Heat tol. rep. } \\
\text { 1A }\end{array}$ & IRA1 & 10589 & 16739 & 14739 \\
$\begin{array}{l}\text { Heat tol. rep. } \\
\text { 1B }\end{array}$ & IRA1 & 10889 & 20689 & 6389 \\
$\begin{array}{l}\text { Heat tol. rep. } \\
2\end{array}$ & IRA1 & 8889 & 2589 & 17289 \\
\hline $\begin{array}{l}\text { Heat tol. rep. } \\
\text { 1A }\end{array}$ & IRA2 & 311 & 3240 & 511 \\
$\begin{array}{l}\text { Heat tol. rep. } \\
\text { 1B }\end{array}$ & IRA2 & 961 & 17670 & 1661 \\
$\begin{array}{l}\text { Heat tol. rep. } \\
2\end{array}$ & IRA2 & 340 & 4190 & 2390 \\
\hline
\end{tabular}

Distances are reported in bases from the MLE to the center of the target gene. The results for IRA1 suggest an additional associated gene; see the text and Figure 5.

Future work could replace our uniform prior over possible causal locus locations with an informative prior that uses conservation data, functional information, or other relevant data types (as in [25]). Other extensions include a more subtle handling of read mapping ambiguities and SNP calling uncertainty. One possibility is to use expected (average) counts under an error-aware probabilistic model instead of hard assignments, which

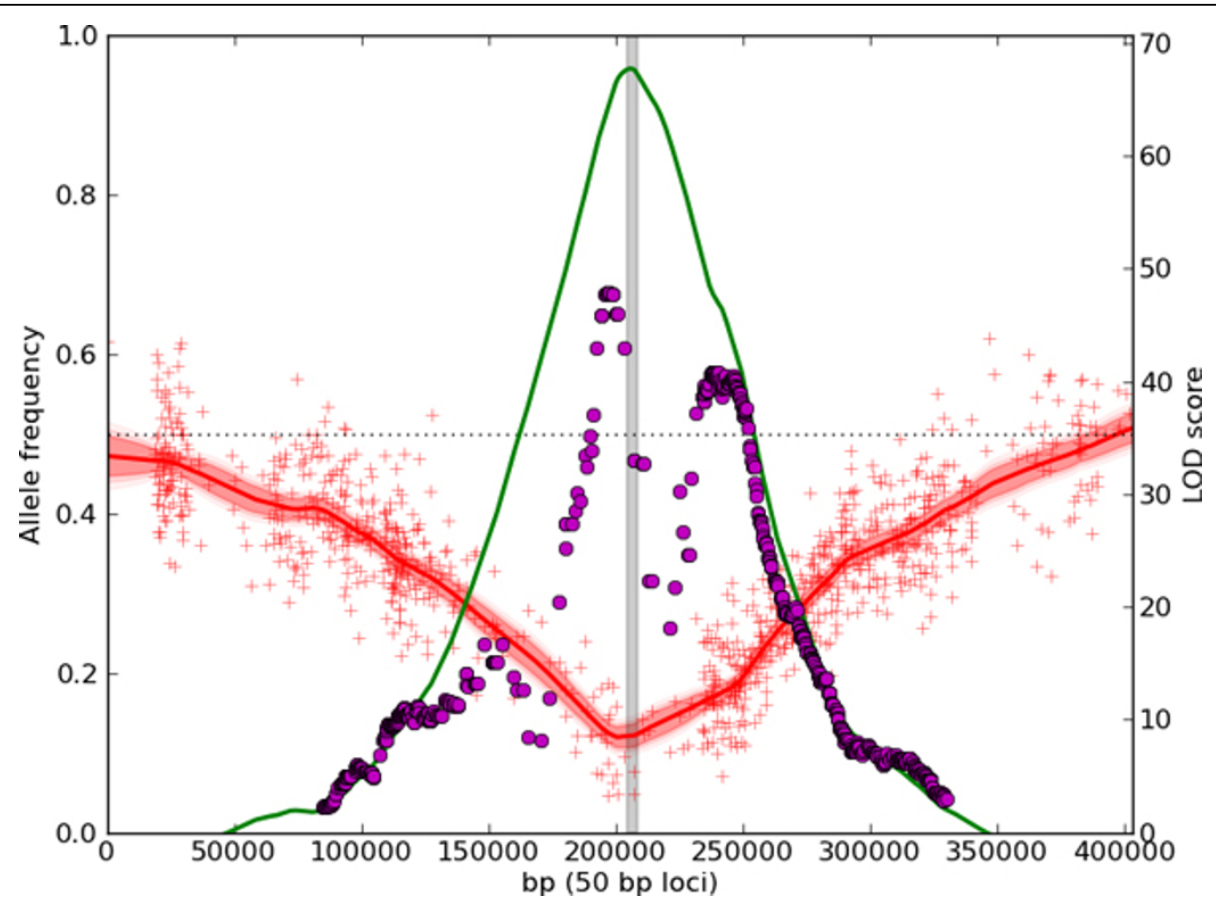

Figure 4 Localization of RAD5 using 4-NQO selected replicate 2. The red line and shaded region show the inferred allele frequencies in the pool using MULTIPOOL, and the red pluses plot the observed allele frequencies from the sequencing data (scale on left axis). Regions without pluses do not have polymorphisms or mappable reads. The magenta dots show LOD scores computed using tests of allele frequencies calculated using $10 \mathrm{~kb}$ sliding windows, while the green line shows the LOD scores calculated using MULTIPOOL (scale on right axis). The gray box shows the position of RAD5, the verified causal gene in the region. 


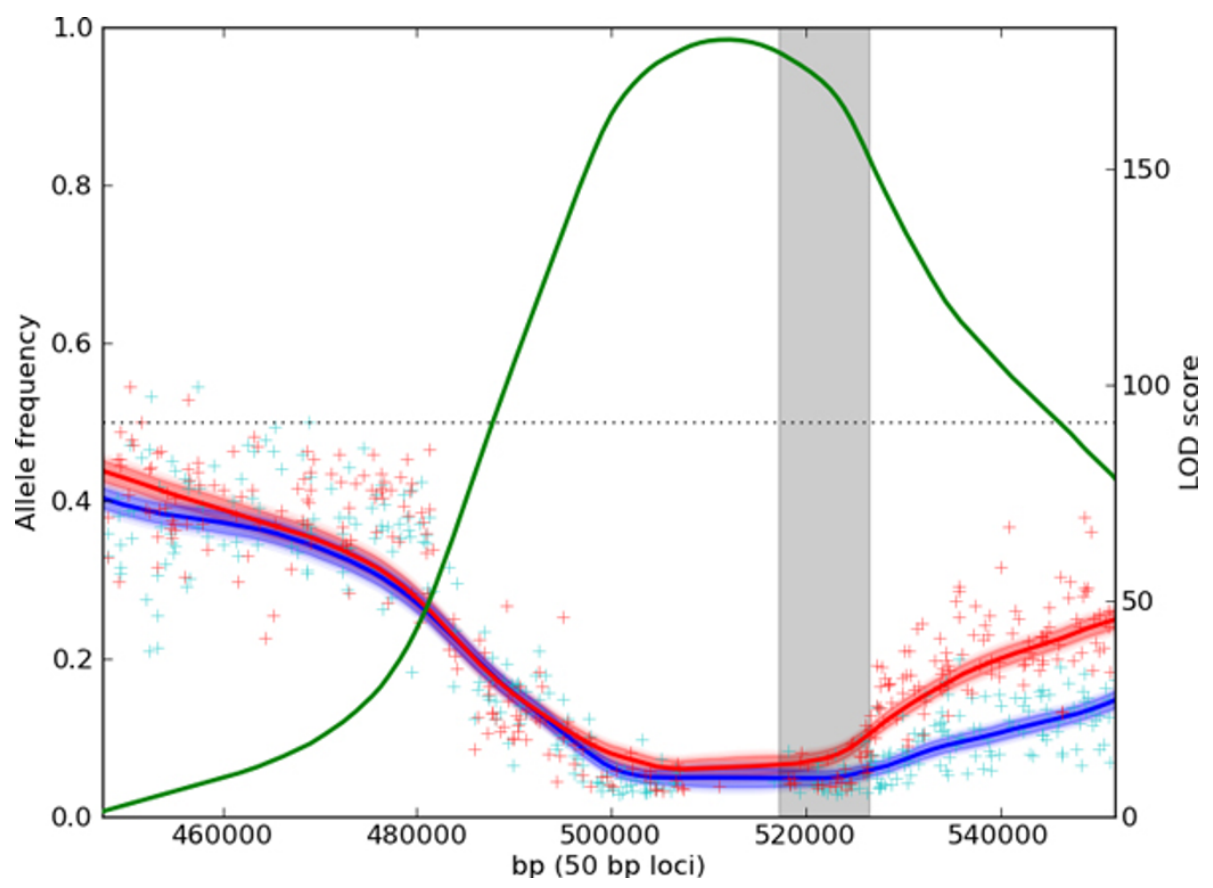

Figure 5 Localization of IRA1 using heat tolerant replicates 1 and 2. The red and blue lines and shaded regions show the inferred allele frequencies in the two replicates using the DBN method, and the pluses plot the observed allele frequencies. The green line shows the LOD scores calculated using the DBN two-pool method. The gray box shows the position of IRAT, the reported and verified association in this region. However, an uncharacterized association upstream of IRA1 may be the cause of the extended range of low allele frequencies and the shifted estimate of the peak location.

should scale gracefully as certainty lowers. This could reduce MultiPool's reliance on a particular aligner and SNP calling strategy.

\section{Abbreviations}

DBN: dynamic Bayesian network; HMM: hidden Markov model; LOD: base 10 logarithm of odds; MLE: maximum likelihood estimate; QTL: quantitative trait locus; RMSE: root mean square error.

\section{Acknowledgements}

We thank lan Ehrenreich for sharing sequencing data and Shaun Mahony for helpful comments on the manuscript. M.D.E. was supported by an NSF Graduate Research Fellowship under grant no. 0645960.

This article has been published as part of BMC Bioinformatics Volume 13 Supplement 6, 2012: Proceedings of the Second Annual RECOMB Satellite Workshop on Massively Parallel Sequencing (RECOMB-seq 2012).

\section{Author details}

'Computer Science and Artificial Intelligence Laboratory, Department of Electrical Engineering and Computer Science, Massachusetts Institute of Technology, Cambridge, MA 02139, USA. "Whitehead Institute for Biomedical Research, Cambridge, MA 02142, USA. ${ }^{3}$ Broad Institute of MIT and Harvard, Cambridge, MA 02142, USA.

\section{Authors' contributions}

MDE and DKG conceived and designed the research. MDE performed the research. MDE and DKG wrote the paper.

\section{Competing interests}

The authors declare that they have no competing interests.

Published: 19 April 2012

\section{References}

1. Michelmore RW, Paran I, Kesseli RV: Identification of markers linked to disease-resistance genes by bulked segregant analysis: a rapid method to detect markers in specific genomic regions by using segregating populations. Proc Natl Acad Sci USA 1991, 88(21):9828-9832.

2. Ehrenreich IM, Torabi N, Jia Y, Kent J, Martis S, Shapiro JA, Gresham D, Caudy AA, Kruglyak L: Dissection of genetically complex traits with extremely large pools of yeast segregants. Nature 2010, 464(7291):1039-1042.

3. Parts L, Cubillos FA, Warringer J, Jain K, Salinas F, Bumpstead SJ, Molin M, Zia A, Simpson JT, Quail MA, Moses A, Louis EJ, Durbin R, Liti G: Revealing the genetic structure of a trait by sequencing a population under selection. Genome Res 2011, 21(7):1131-1138.

4. Wenger JW, Schwartz K, Sherlock G: Bulk segregant analysis by highthroughput sequencing reveals a novel xylose utilization gene from Saccharomyces cerevisiae. PLoS Genet 2010, 6(5):e1000942.

5. Andolfatto P, Davison D, Erezyilmaz D, Hu TT, Mast J, Sunayama-Morita T, Stern DL: Multiplexed shotgun genotyping for rapid and efficient genetic mapping. Genome Res 2011, 21(4):610-617.

6. Schneeberger K, Ossowski S, Lanz C, Juul T, Petersen AH, Nielsen KL, Jørgensen J, Weigel D, Andersen SU: SHOREmap: simultaneous mapping and mutation identification by deep sequencing. Nat Methods 2009, 6(8):550-551.

7. Calvo SE, Tucker EJ, Compton AG, Kirby DM, Crawford G, Burtt NP, Rivas M, Guiducci C, Bruno DL, Goldberger OA, Redman MC, Wiltshire E, Wilson CJ, Altshuler D, Gabriel SB, Daly MJ, Thorburn DR, Mootha VK: Highthroughput, pooled sequencing identifies mutations in NUBPL and FOXRED1 in human complex I deficiency. Nat Genet 2010, 42(10):851-858.

8. Borevitz JO, Liang D, Plouffe D, Chang H, Zhu T, Weigel D, Berry CC, Winzeler $\mathrm{E}$, Chory J: Large-scale identification of single-feature polymorphisms in complex genomes. Genome Res 2003, 13(3):513-523.

9. Hazen SP, Borevitz JO, Harmon FG, Pruneda-Paz JL, Schultz TF, Yanovsky MJ, Liljegren SJ, Ecker JR, Kay SA: Rapid array mapping of circadian clock and developmental mutations in Arabidopsis. Plant Physiol 2005, 138(2):990-997. 
10. Brauer MJ, Christianson CM, Pai DA, Dunham MJ: Mapping novel traits by array-assisted bulk segregant analysis in Saccharomyces cerevisiae. Genetics 2006, 173(3):1813-1816.

11. Sham P, Bader JS, Craig I, O'Donovan M, Owen M: DNA Pooling: a tool for large-scale association studies. Nat Rev Genet 2002, 3(11):862-871.

12. Jawaid A, Bader JS, Purcell S, Cherny SS, Sham P: Optimal selection strategies for QTL mapping using pooled DNA samples. Eur J Hum Genet 2002, 10(2):125-132.

13. Macgregor S, Zhao ZZ, Henders A, Nicholas MG, Montgomery GW, Visscher PM: Highly cost-efficient genome-wide association studies using DNA pools and dense SNP arrays. Nucleic Acids Res 2008, 36(6):e35.

14. Degner JF, Marioni JC, Pai AA, Pickrell JK, Nkadori E, Gilad Y, Pritchard JK Effect of read-mapping biases on detecting allele-specific expression from RNA-sequencing data. Bioinformatics 2009, 25(24):3207-3212.

15. McPeek MS, Strahs A: Assessment of linkage disequilibrium by the decay of haplotype sharing, with application to fine-scale genetic mapping. Am J Hum Genet 1999, 65(3):858-875.

16. Morris AP, Whittaker JC, Balding DJ: Bayesian fine-scale mapping of disease loci, by hidden Markov models. Am J Hum Genet 2000, 67:155-169.

17. Johnson T: Bayesian method for gene detection and mapping, using a case and control design and DNA pooling. Biostatistics 2007, 8(3):546-565.

18. Homer N, Tembe WD, Szelinger S, Redman M, Stephan DA, Pearson JV, Nelson SF, Craig D: Multimarker analysis and imputation of multiple platform pooling-based genome-wide association studies. Bioinformatics 2008, 24(17):1896-1902.

19. Li H, Durbin R: Fast and accurate long-read alignment with BurrowsWheeler transform. Bioinformatics 2010, 26(5):589-595.

20. Li H, Handsaker B, Wysoker A, Fennell T, Ruan J, Homer N, Marth G, Abecasis G, Durbin R: The Sequence Alignment/Map format and SAMtools. Bioinformatics 2009, 25(16):2078-2079.

21. Bishop CM: Pattern Recognition and Machine Learning Springer; 2007.

22. Murphy K: Filtering, Smoothing and the Junction Tree Algorithm. University of California, Berkeley Technical Report 1999, http://www.cs.ubc.ca/ $\sim$ murphyk/Papers/smooth.ps.gz.

23. Ghahramani, Hinton GE: Parameter estimation for linear dynamical systems. University of Toronto Technical Report 1996, 6(CRG-TR-96-2):1-6, http://www.cs.toronto.edu/ hinton/absps/tr-96-2.pdf.

24. Mancera E, Bourgon R, Brozzi A, Huber W, Steinmetz LM: High-resolution mapping of meiotic crossovers and non-crossovers in yeast. Nature 2008, 454(7203):479-485

25. Lee S, Dudley AM, Drubin D, Silver PA, Krogan NJ, Pe'er D, Koller D: Learning a prior on regulatory potential from eQTL data. PLOS Genet 2009, 5:e1000358.

doi:10.1186/1471-2105-13-S6-S8

Cite this article as: Edwards and Gifford: High-resolution genetic mapping with pooled sequencing. BMC Bioinformatics 2012 13(Suppl 6): S8.

\section{Submit your next manuscript to BioMed Central and take full advantage of:}

- Convenient online submission

- Thorough peer review

- No space constraints or color figure charges

- Immediate publication on acceptance

- Inclusion in PubMed, CAS, Scopus and Google Scholar

- Research which is freely available for redistribution 